\title{
Frozen Section Intraoperatively is it Adequately Accurate in Endometrial Carcinoma Cases?
}

\author{
Khaled Fathy Helal ${ }^{1 *}$ and Mai Mohamed Abdelwahab ${ }^{2}$ \\ ${ }^{1}$ Department of Obstetrics and Gynecology, Zagazig University, Egypt \\ ${ }^{2}$ Department of pathology, Zagazig University, Egypt
}

Submission: May 13, 2019 ; Published: June 06, 2019

*Corresponding author: Khaled Fathy Helal, Department of Obstetrics and Gynecology, Zagazig University, Egypt

\begin{abstract}
Background: Frozen section is one of the basic diagnostic tools implemented intraoperatively to elucidate the requirement of lymph node dissection, however diagnostic accuracy is a matter of debate among various research groups in oncological centers all over the globe.

Methodology: A retrospective clinical research trial that recruited 90 research study subjects having endometrial carcinoma in which all recruited subjects Had histological grading and invasion level of muscular tissue planes assessed by usage of intraoperative frozen section investigative tool from February 2016 till January 2019.

Results: The comparative analyses of the Risk of Lymph-Node Metastasis between the Preoperative, frozen section and Permanent-Section Diagnoses in the 90 Patients with Endometrial Carcinoma in which as regards preoperative diagnosis in comparison to permanent section diagnosis concerning low risk, intermediate risk, high risk, total accuracy was 57.69\%, 50.00\%, 53.33\%, 53.3\% consecutively, whereas frozen section diagnosis accuracy as regards low risk, intermediate risk, high risk, total accuracy was $88.46 \%, 73.53 \%, 66.67 \%, 75.6 \%$ consecutively.

Conclusions and recommendations: The current research study have shown the superiority of frozen section over MRI and various perioperative assessment tools in evaluating accurately invasiveness, grade and lymph node disease, however the current research study findings should be interpreted with caution as other modes for diagnosis are gaining privilege over cosurgical practice such as sentinel lymph node mapping.
\end{abstract}

\section{Introduction}

Cancer endometrium is basically managed by total abdominal hysterectomy and bilateral salpingo-oophorectomy, however the challenging surgical decision is the pelvic lymph adenectomy that could be required in advanced stages of the disease. The challenge arises since routine performance of pelvic lymph adenectomy is not required in all cases [1,2]. Frozen section is one of the basic diagnostic tools implemented intraoperatively to elucidate the requirement of lymph node dissection, however diagnostic accuracy is a matter of debate among various research groups in oncological centers all over the globe, besides it is crucial to determine the staging of the disease in a meticulous manner since that would elucidate the best management protocol after commencement of surgery. Side effects arising from total pelvic lymphadnectomy such as lower limb lymph edema is one of the corner stone issues that could affect the 5 year patient survival rate $[3,4]$. Researchers all over the globe are having increased interest in revealing the best diagnostic tool effective intraoperatively to elucidate the presence of metastasis within the lymph nodes as that would affect the quality of care and extent of surgery required, even post-operative chemo or radio therapy would be determined by the lymph node affection issue $[5,6]$.
Para-aortic lymphadenectomy and omentectomy are also, like the pelvic nodes, if the histological grade is endometrioid G1 or G2 and muscular invasion is shallow, the metastasis probability is low and the value gained by the patient from para-aortic lymphadenectomy and omentectomy is small $[7,8]$. Muscular invasion degree and histological grading denotes and clarifies for the gyne-oncologic surgeon whether lymphadenectomy and omentectomy are required to be performed. Prior research studies have revealed and displayed that, the diagnostic accuracy of muscular invasion by implementing magnetic resonance imaging (MRI), transvaginal sonography and intraoperative macroscopic observation was $54-90 \%, 73-87 \%$ and $74-91 \%$, consecutively interestingly the precision of determining histologic grading by usage of endometrial biopsy preoperatively was mentioned by various research groups of investigators to be around 35\% to 97\% [9-11].

Clinical diagnoses based on evaluating degree of invasiveness of muscular tissue planes and histological grading by usage of MRI preoperatively and biopsy could result in over underestimation of lymph-node metastasis risk. Since over grading of the disease extension causes performance of needless lymph- 
adenectomy, that raises time period of surgical performance and increase the rate of complications that are correlated to the procedure. Underestimation raises the clinical risk recurrence of the disease from affected lymph nodes after completion of surgical procedure and could cause missed crucial postoperative management pathway in the form of chemo or radio therapy according to surgical case scenario. Endometrial cancer even at early stages requires multidisciplinary management team that aids in clarification of the best management pathway that is tailored according to the patient needs and intraoperative findings [12-14].

Usage of intraoperative frozen section for diagnosis could reduce unrequired lymphadenectomy and on the other hand could raise the rate of persistence of diseased lymph nodes. Various researchers in the field of gyne-oncology have shown that frozen section is a useful diagnostic tool in determining histological grade and muscular invasion intraoperatively in comparison and contrast to routine preoperative assessment protocols $[15,16]$.

\section{Methodology}

A retrospective clinical research trial that recruited 90 research study subjects having endometrial carcinoma in which all recruited subjects Had histological grading and invasion level of muscular tissue planes assessed by usage of intraoperative frozen section investigative tool from February 2016 till January 2019 at Department of Obstetrics and Gynecology, Zagazig University, Egypt. Biopsy specimens preoperatively was obtained by usage of endometrial curettage, Preoperative invasiveness of muscular tissue planes have been assessed by using MRI. Cases have been categorized into three categories: endometrioid G1, endometrioid G2 and high-grade malignancy (endometrioid G3, serous, clear cell), and preoperative evaluation and frozen section diagnosis accuracy have been statistically calculated. As regards invasiveness of muscular tissue planes cases have been categorized into three categories: no muscular invasion, below $1 / 2$ wall invasion and $\geq 1 / 2$ wall invasion, and the accuracy of the preoperative assessment and frozen-section diagnosis have been statistically calculated. As regards lymph nodes disease risk cases recruited were categorized into three risk groups (low, intermediate and high) according to the risk for lymph-node metastasis.

Cases within the low-risk group had no muscular invasion and the histological grade was G1 or G2. Cases within the intermediate- risk group had below $1 / 2$ wall muscular invasion and the histological grade was G1 or G2. Cases within the high-risk group had $\geq 1 / 2$ muscular wall invasion or a histologically high grade. Consequently, statistical calculation of preoperative assessment and frozen-section diagnosis accuracy levels was determined.

\section{Statistical Analysis}

Data were collected, revised, coded and entered to the Statistical Package for Social Science (IBM SPSS) version 23 and presented as numbers and percentages. McNemar test was used to compare the accuracies of the preoperative evaluation and frozen-section diagnoses. The confidence interval was set to $95 \%$ and the margin of error accepted was set to $5 \%$.

\section{Result}

Table 1: Basic characteristics of the total research study cohort.

\begin{tabular}{|c|c|}
\hline Age (years) & $\mathbf{5 4 . 5} \pm 3.7$ \\
\hline Stage, no. (\%) & \\
\hline IA & $52(57.8 \%)$ \\
\hline IB & $21(23.3 \%)$ \\
\hline II & $2(2.2 \%)$ \\
\hline IIIA & $5(5.6 \%)$ \\
\hline IIIB & $1(1.1 \%)$ \\
\hline IIIC1 & $4(4.4 \%)$ \\
\hline IIIC2 & $5(5.6 \%)$ \\
\hline Grade, no. (\%) & \\
\hline G1 & $58(64.4 \%)$ \\
\hline G2 & $32(35.6 \%)$ \\
\hline Risk classification, no. $(\%)$ & $8(8.9 \%)$ \\
\hline High grade & $26(28.9 \%)$ \\
\hline Low risk & $26(28.9 \%)$ \\
\hline Intermediate risk & $30(33.3 \%)$ \\
\hline High risk & \\
\hline
\end{tabular}

Table 1 reveals and displays that the mean age +/-SD $=54.5 \pm 3.7$ years, 52 cases representing $57.8 \%$ of the cohort had stage IA disease, 21 cases representing $23.3 \%$ of the total cohort had stage IB, whereas 58 cases had G1 representing $64.4 \%$ and 32 cases had G2 representing $35.65 \%$ of the total cohort additionally according to risk classification 30 cases have been classified as high risk representing $33.3 \%$ of cases, 26 cases in each category low and intermediate risk representing each $28.9 \%$ of the total number of cases, High grade category was present in 8 cases representing $8.9 \%$ of cases total number.

Table 2: Comparison of the Tumor Grade between the Preoperative, frozen section and Permanent-Section Diagnoses in the 90 Patients with Endometrial Carcinoma.

\begin{tabular}{|c|c|c|c|c|c|c|c|}
\hline \multirow{2}{*}{ Permanent-Section Diagnosis } & \multicolumn{5}{|c|}{ Preoperative Diagnosis } & \multirow{2}{*}{ Total } & \multirow{2}{*}{ Accuracy } \\
\cline { 2 - 7 } & Unknown & No Tumor & G1 & G2 & High Grade & & 54 \\
\hline G1 & 4 & 3 & 44 & 3 & 0 & $81.48 \%$ \\
\hline G2 & 4 & 2 & 9 & 11 & 0 & 26 & $42.31 \%$ \\
\hline High grade & 1 & 1 & 3 & 1 & 4 & 10 & $40.00 \%$ \\
\hline Total & 9 & 6 & 56 & 15 & 4 & 90 & $65.60 \%$ \\
\hline
\end{tabular}




\section{Journal of Gynecology and Women's Health}

\begin{tabular}{|c|c|c|c|c|c|c|c|}
\hline \multirow{2}{*}{ Permanent-Section Diagnosis } & \multicolumn{5}{|c|}{ Frozen-Section Diagnosis } & \multirow{2}{*}{ Total } & \multirow{2}{*}{ Accuracy } \\
\cline { 2 - 7 } & Unknown & No Tumor & G1 & G2 & High Grade & \\
\hline G1 & 1 & 3 & 46 & 3 & 0 & 53 & $86.79 \%$ \\
\hline G2 & 1 & 0 & 13 & 13 & 1 & 28 & $46.43 \%$ \\
\hline High grade & 0 & 0 & 3 & 3 & 3 & 9 & $33.33 \%$ \\
\hline Total & 2 & 3 & 62 & 19 & 4 & 90 & $68.90 \%$ \\
\hline
\end{tabular}

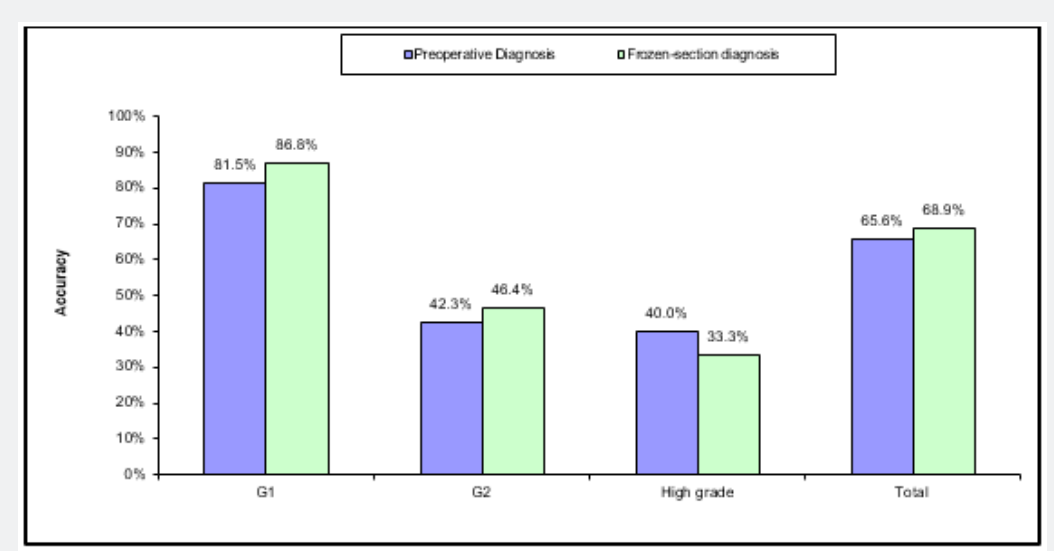

Figure 1: Comparison of the Tumor Grade between the Preoperative, frozen section and Permanent-Section Diagnoses in the 90 Patients with Endometrial Carcinoma.

Table 2 and Figure 1 reveal and display the comparative analysis between preoperative, frozen section and Permanent-Section Diagnoses in the 90 cases having Endometrial Carcinoma in which in comparison to permanent section diagnosis preoperative diagnosis at G1,G2, high grade, total accuracy was $81.48 \%$,

Table 3: Comparison of the Tumor Grade between the Preoperative, frozen section and Permanent-Section Diagnoses in the 90 Patients with Endometrial Carcinoma.

\begin{tabular}{|c|c|c|c|c|c|c|c|}
\hline \multirow{2}{*}{ Permanent-Section Diagnosis } & \multicolumn{5}{|c|}{ Preoperative MRI Diagnosis } & \multirow{2}{*}{ Total } & \multirow{2}{*}{ Accuracy } \\
\hline & Unknown & Invasion present & No invasion & $<1 / 2$ & $>1 / 2$ & & \\
\hline No invasion & 0 & 1 & 19 & 9 & 1 & 30 & $63.33 \%$ \\
\hline$<1 / 2$ & 1 & 3 & 8 & 17 & 2 & 31 & $54.84 \%$ \\
\hline$>1 / 2$ & 2 & 2 & 2 & 5 & 20 & 31 & $64.52 \%$ \\
\hline Total & 3 & 5 & 28 & 31 & 22 & 92 & $60.90 \%$ \\
\hline \multirow{2}{*}{ Permanent-Section Diagnosis } & \multicolumn{5}{|c|}{ Frozen-Section Diagnosis } & \multirow{2}{*}{ Total } & \multirow{2}{*}{ Accuracy } \\
\hline & Unknown & Invasion Present & No Invasion & $<1 / 2$ & $>1 / 2$ & & \\
\hline No invasion & 1 & 0 & 25 & 2 & 0 & 28 & $89.29 \%$ \\
\hline$<1 / 2$ & 0 & 0 & 6 & 24 & 1 & 31 & $77.42 \%$ \\
\hline$>1 / 2$ & 0 & 1 & 2 & 9 & 19 & 31 & $61.29 \%$ \\
\hline Total & 1 & 1 & 34 & 35 & 20 & 90 & $75.60 \%$ \\
\hline
\end{tabular}

Table 3 and Figure 2 reveal and display the comparison of the Muscular Invasion between the Preoperative MRI, frozen section and Permanent-Section Diagnoses in the 90 Patients with Endometrial Carcinoma, in which in comparison to permanent section diagnosis Preoperative MRI Diagnosis as regards no invasion, $<1 / 2,>1 / 2$, total accuracy was $63.33 \%, 54.84 \%, 64.52 \%$, $60.9 \%$ consecutively, whereas frozen section diagnosis in comparison to permanent section diagnosis as regards no invasion, $<1 / 2,>1 / 2$, total accuracy was $89.29 \%, 77.42 \%, 61.29 \%, 75.6 \%$ consecutively.
$42.31 \%, 40.00 \%, 65.6 \%$ consecutively .whereas in comparison G2, high grade, total accuracy was 86.79\%, 46.43\%, 33.33\%, $68.9 \%$ consecutively. to permanent section diagnosis frozen section diagnosis at G1,

Table 4 and Figure 3 reveals and displays the comparative analyses of the Risk of Lymph-Node Metastasis between the Preoperative, frozen section and Permanent-Section Diagnoses in the 90 Patients with Endometrial Carcinoma in which as regards preoperative diagnosis in comparison to permanent section diagnosis concerning low risk, intermediate risk, high risk, total accuracy was 57.69\%, 50.00\%, 53.33\%, 53.3\% consecutively, whereas frozen section diagnosis accuracy as regards low risk, intermediate risk, high risk, total accuracy was $88.46 \%, 73.53 \%$, $66.67 \%, 75.6 \%$ consecutively . 


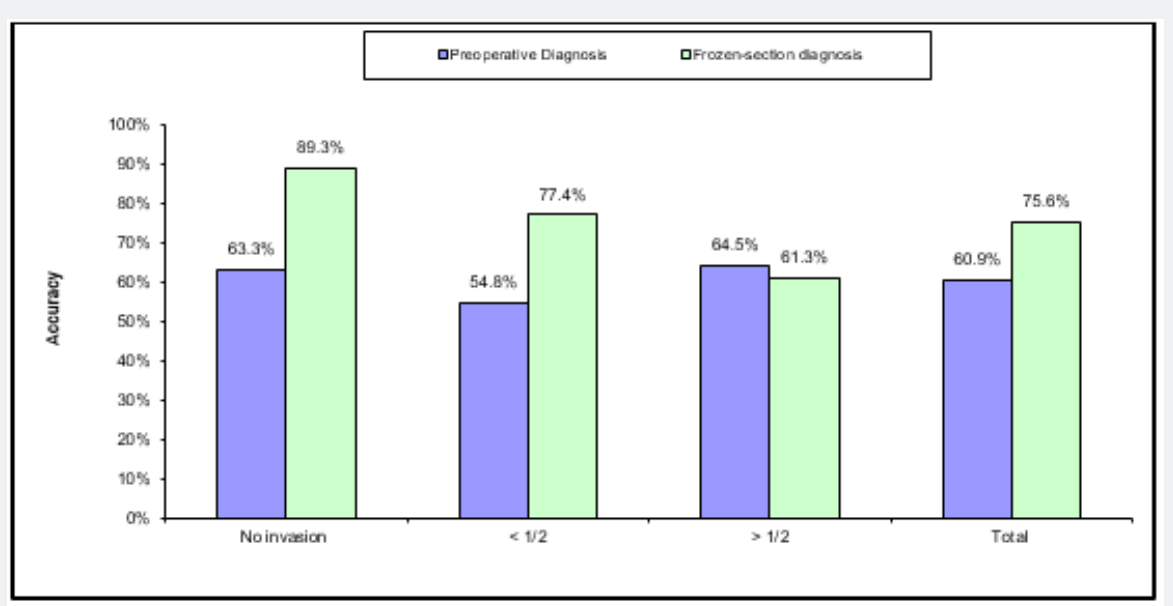

Figure 2: Comparison of the Muscular Invasion between the Preoperative MRI, frozen section and Permanent-Section Diagnoses in the 90 Patients with Endometrial Carcinoma.

Table 4: Comparison of the Risk of Lymph-Node Metastasis between the Preoperative, frozen section and Permanent-Section Diagnoses in the 90 Patients with Endometrial Carcinoma.

\begin{tabular}{|c|c|c|c|c|c|c|}
\hline \multirow{2}{*}{ Permanent-Section Diagnosis } & \multicolumn{4}{|c|}{ Preoperative Diagnosis } & \multirow{2}{*}{ Total } & \multirow{2}{*}{ Accuracy } \\
\hline & Unknown & Low Risk & Intermediate Risk & High Risk & & \\
\hline Low risk & 3 & 15 & 7 & 1 & 26 & $57.69 \%$ \\
\hline Intermediate risk & 6 & 8 & 17 & 3 & 34 & $50.00 \%$ \\
\hline High risk & 7 & 4 & 3 & 16 & 30 & $53.33 \%$ \\
\hline Total & 16 & 27 & 27 & 20 & 90 & $53.30 \%$ \\
\hline \multirow{2}{*}{ Permanent-Section Diagnosis } & \multicolumn{4}{|c|}{ Frozen-Section Diagnosis } & \multirow{2}{*}{ Total } & \multirow{2}{*}{ Accuracy } \\
\hline & Unknown & Low Risk & Intermediate Risk & High Risk & & \\
\hline Low risk & 1 & 23 & 2 & 0 & 26 & $88.46 \%$ \\
\hline Intermediate risk & 0 & 7 & 25 & 2 & 34 & $73.53 \%$ \\
\hline High risk & 2 & 2 & 6 & 20 & 30 & $66.67 \%$ \\
\hline Total & 3 & 31 & 35 & 21 & 90 & $75.60 \%$ \\
\hline
\end{tabular}

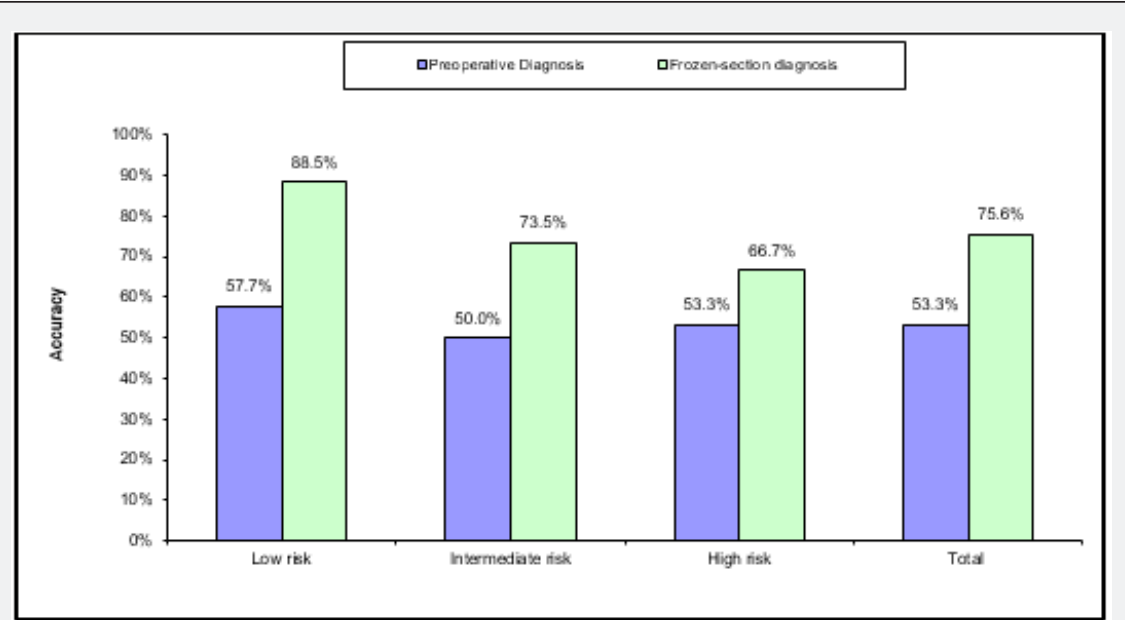

Figure 3: Comparison of the Risk of Lymph-Node Metastasis between the Preoperative, frozen section and Permanent-Section Diagnoses in the 90 Patients with Endometrial Carcinoma.

\section{Discussion}

Endometrial carcinoma a challenging oncological surgical procedure as regards the extent of the procedure that should be performed ,various anatomical ,diagnostic and medical challenging issues arise .Frozen section diagnosis of the disease extent is one of the diagnostic tools that are implemented to reveal the extent of lymph nodes affected by the disease $[17,18]$. 
The current research study findings revealed the following in which comparative analysis between preoperative, frozen section and Permanent-Section Diagnoses in the 90 cases having Endometrial Carcinoma in which in comparison to permanent section diagnosis preoperative diagnosis at G1, G2, high grade, total accuracy was $81.48 \%, 42.31 \%, 40.00 \%, 65.6 \%$ consecutively. whereas in comparison to permanent section diagnosis frozen section diagnosis at G1, G2, high grade, total accuracy was $86.79 \%, 46.43 \%, 33.33 \%, 68.9 \%$ consecutively. Besides the current research results revealed that preoperative MRI Diagnosis as regards no invasion, $<1 / 2,>1 / 2$, total accuracy was $63.33 \%, 54.84 \%, 64.52 \%, 60.9 \%$ consecutively, whereas frozen section diagnosis in comparison to permanent section diagnosis as regards no invasion, $<1 / 2,>1 / 2$, total accuracy was $89.29 \%$, $77.42 \%, 61.29 \%, 75.6 \%$ consecutively. Finally preoperative diagnosis in comparison to permanent section diagnosis concerning low risk, intermediate risk, high risk, total accuracy was $57.69 \%$, $50.00 \%, 53.33 \%$, 53.3\% consecutively, whereas frozen section diagnosis accuracy as regards low risk, intermediate risk, high risk, total accuracy was $88.46 \%, 73.53 \%, 66.67 \%, 75.6 \%$ consecutively.

Determination of nodal metastasis risk and lymphadnectomy requirement is liable for diagnostic errors. Using alternative, sentinel lymph node mapping and ultra-staging besides frozen section diagnosis are implemented in endometrial carcinoma surgical management guidelines. Interestingly prior research findings justify the usage of sentinel lymph node biopsy in preliminary endometrial carcinaoma surgery as a counter balance to intra frozen section diagnostic errors, reducing their negative impact on patient care as revealed by various research groups of investigators $[19,20]$.

It was proposed and suggested by various gyneoncological surgeons to conduct Sentinel lymph node mapping for all endometrial carcinoma cases. Cases misclassified by intraoperative frozen section, sentinel lymph node is an effective supplement that provides useful prognostic information. A prior research study have shown that $22 \%$ of cases recruited were misdiagnosed by frozen section, and that those errors could have caused 14 patients receiving the wrong staging procedure e.g. unnecessary lymphadenectomy, 10 cases would have omitted crucial lymph node evaluation $[1,3,9]$.

Diagnosing high-grade tumor types (e.g. serous, clear cell, sarcoma) on frozen section could be a challenging oncological issue, even in presence of preoperative diagnoses have been made available. It was revealed and displayed by various prior research teams of investigators that intra operative frozen section sensitivity was statistically significantly lower for G3 tumors (78\%) in Comparison to G1 and G2 (89.3\% and 91.1\% Consecutively). [2,7,11].

Histopathologists could easily misdiagnose high-grade tumors. Furthermore prior investigators have mentioned an interested fact that $60 \%$ of frozen section errors arise due to sampling and $40 \%$ to pathologist interpretation issues however diagnostic skills of histopathologists could be enhanced by focused training, on the other hand intraoperative frozen section errors appear to be unavoidable due to intrinsic restrictions and time limits. Interestingly a prior research team of investigators Have used sentinel lymph node in all recruited cases and ignored widespread frozen section diagnostic histopathological approach, limiting its usage to cases having failed sentinel lymph node mapping $[13,15,19]$.

\section{Conclusion and Recommendations}

The current research study have shown the superiority of frozen section over MRI and various perioperative assessment tools in evaluating accurately invasiveness, grade and lymph node disease ,however the current research study findings should be interpreted with caution as other modes for diagnosis are gaining privilege in oncosurgical practice such as sentinel lymph node mapping .Future research efforts are required to be multicentric in fashion putting in consideration comparative analysis of all used diagnostic tools preoperatively and intraoperatively available for the managing team that would future clinical guideline implementation.

\section{References}

1. Furukawa N, Takekuma M, Takahashi N, Hirashima Y (2010) Intraoperative evaluation of myometrial invasion and histological type and grade in endometrial cancer: diagnostic value of frozen section. Arch Gynecol Obstet 281(5): 913-917.

2. Emlik D, Kiresi D, Ozdemir S, Celik C, Karakose S, et al. (2010) Preoperative assessment of myometrial and cervical invasion in endometrial carcinoma: comparison of multi-section dynamic MR imaging using a three dimensional FLASH technique and T2-weighted MR imaging. J Med Imaging Radiat Oncol 54(3): 202-210.

3. Ugaki H, Kimura T, Miyatake T, Ueda Y, Yoshino K, et al. (2011) Intraoperative frozen section assessment of myometrial invasion and histology of endometrial cancer using the revised FIGO staging system. Int J Gynecol Cancer 21(7): 1180-1184.

4. Kisu I, Banno K, Lin LY, Ueno A, Abe T, et al. (2013) Preoperative and intraoperative assessment of myometrial invasion in endometrial cancer: comparison of magnetic resonance imaging and frozen sections. Acta Obstet Gynecol Scand 92(5): 525-535.

5. Gallego JC, Porta A, Pardo MC, Fernandez C (2014) Evaluation of myometrial invasion in endometrial cancer: comparison of diffusion-weighted magnetic resonance and intraoperative frozen sections. Abdom Imaging 39(5): 1021-1026.

6. Akbayir O, Corbacioglu A, Numanoglu C, Goksedef BP, Guraslan H, et al. (2012) Combined use of preoperative transvaginal ultrasonography and intraoperative gross examination in the assessment of myometrial invasion in endometrial carcinoma. Eur J Obstet Gynecol Reprod Biol 165(2): 284-288.

7. Ozturk E, Dikensoy E, Balat O, Ugur MG, Aydin A, et al. (2012) Intraoperative frozen section is essential for assessment of myometrial invasion but not for histologic grade confirmation in endometrial cancer: a ten-year experience. Arch Gynecol Obstet. 285(5): 1415-1419.

8. Savelli L, Testa AC, Mabrouk M, Zannoni L, Ludovisi M, et al. (2012) A prospective blinded comparison of the accuracy of transvaginal sonography and frozen section in the assessment of myometrial invasion in endometrial cancer. Gynecol Oncol 124(3): 549-552. 
9. Sethasathien P, Charoenkwan K, Siriaunkgul S (2014) Accuracy of intraoperative gross examination of myometrial invasion in stage I-II endometrial cancer. Asian Pac J Cancer Prev 15(17): 7061-7064.

10. Marcickiewicz J, Sundfeldt K (2011) Accuracy of intraoperative gross visual assessment of myometrial invasion in endometrial cancer. Acta Obstet Gynecol Scand 90(8): 846-851.

11. Wang X, Li L, Cragun JM, Chambers SK, Hatch KD, et al. (2016) Assessment of the role of intraoperative frozen section in guiding surgical staging for endometrial cancer. Int J Gynecol Cancer 26(5): 918-923.

12. Acikalin A, Gumurdulu D, Bagir EK, Torun G, Guzel AB, et al. (2015) The guidance of intraoperative frozen section for staging surgery in endometrial carcinoma: frozen section in endometrial carcinoma. Pathol Oncol Res 21(1): 119-122.

13. Karabagli P, Ugras S, Yilmaz BS, Celik C (2015) The evaluation of reliability and contribution of frozen section pathology to staging endometrioid adenocarcinomas. Arch Gynecol Obstet 292(2): 391-397.

14. Stephan JM, Hansen J, Samuelson M, McDonald M, Chin Y, et al. (2014) Intra-operative frozen section results reliably predict final pathology in endometrial cancer. Gynecol Oncol 133(3): 499-505.
15. Turan T, Oguz E, Unlubilgin E, Tulunay G, Boran N, et al. (2013) Accuracy of frozen-section examination for myometrial invasion and grade in endometrial cancer. Eur J Obstet Gynecol Reprod Biol 167(1): 90-95.

16. Kumar S, Medeiros F, Dowdy SC, Keeney GL, Bakkum- Gamez JN, et al (2012) A prospective assessment of the reliability of frozen section to direct intraoperative decision making in endometrial cancer. Gynecol Oncol 127(3): 525-531.

17. Bogani G, Dowdy SC, Cliby WA, Ghazi F, Rossetti D, et al. (2014) Role of pelvic and paraaortic lymphadenectomy in endometrial cancer: current evidence. J Obstet Gynaecol Res 40(2): 301-311.

18. Khoury-Collado F, Clair CS, Abu-Rustum NR (2016) Sentinel lymph node mapping in endometrial cancer: an update. Oncologist 21(4): 461-466.

19. Kim CH, Soslow RA, Park KJ, Barber EL, Khoury-Collado F, et al. (2013) Pathologic ultrastaging improves micrometastasis detection in sentinel lymph nodes during endometrial cancer staging. Int J Gynecol Cancer 23(5): 964-970.

20. Naoura I, Canlorbe G, Bendifallah S, Marcos Ballester, Emile Daraï, et al. (2015) Relevance of sentinel lymph node procedure for patients with high-risk endometrial cancer. Gynecol Oncol 136(1): 60-64.

\section{Your next submission with Juniper Publishers will reach you the below assets}

- Quality Editorial service

- Swift Peer Review

- Reprints availability

- E-prints Service

- Manuscript Podcast for convenient understanding

- Global attainment for your research

- Manuscript accessibility in different formats

( Pdf, E-pub, Full Text, Audio)

- Unceasing customer service

Track the below URL for one-step submission

https://juniperpublishers.com/online-submission.php 